\title{
Survey of aminoglycoside acetyl transferase genes in multi-drug resistance acinetobacter
}

\begin{abstract}
Acinetobacter baumannii is a gram-negative, non-fermenting cocobacills that its species are opportunistic pathogens and cause nosocomial infections. Bacteria achieve their resistance to the antibiotics through three mechanisms. aminoglycoside acetyl transferase are member of GCN5 super family as an AMEs for example AAC(3) and AAC(6) N acetylate aminoglycoside on the amine grope on position 3 and 6 amino hexose respectively. In this is study we are survey present of AAC $(3,6)$ gen in Acinetobacter baumannii isolated from Iranian hospital patient. A total of 43 non-duplicated Acinetobacter clinical isolates were collected from the Tehran hospital in 2016. DNA extraction carried out by gram negative DNA extraction kit. Two target gens and their primers used for PCR amplification. Result show that aac (3) IIa gene with $740 \mathrm{bp}$ was $68.4 \%$ and in gentamicin resistant strain was $66.9 \%$ aac (6) Ib gene with $482 \mathrm{bp}$ present in $76 \%$ of resistant strain (43/33) and in amikacin resistant strain was $72.2 \%$ and in gentamicin resistant strain was $70.6 \%$. Preview study showed presence of high variety of resistance gene especially aminoglycoside. This variety explains that other genes may be have role in Acinetobacter resistance for aminoglycoside. It believes that the ability of this pathogen to harbor diverse genetic elements parallels the experience with $P$. aeruginosa. Genome wide analysis will provide critical insights into this ability.
\end{abstract}

Keywords: acinetobacter, aminoglycoside acetyl transferase, aminoglycoside, pcr
Volume 4 Issue I - 2018

Saleh Soleimani,' Samira Vaziri,', Mona Afrasiyabi, ${ }^{3}$ Habibollah Nazem,' Mohhamd Fazilati,' Seyyed mohhamad Atyabi ${ }^{2}$

'Department of biology, Payamenoor university, Iran ${ }^{2}$ Department of pilot biotechnology, pasteure institute of Iran, Iran

${ }^{3}$ Pharmaceutical sciences branch, Islamic azad university, Iran

Correspondence: Seyyed mohammad atyabi, Department of pilot biotechnology, Pasteure institute of Iran, Iran, Tel +989122061565, Email mohammadatyabi@yahoo.com

Received: December 29, 2017| Published: January 29, 2018

\section{Introduction}

Acinetobacter baumannii is a gram-negative, non fermenting cocobacills that its species are opportunistisic pathogens and cause nosocomial infections among patients in intensive care unit (ICUs). This bacteria cause various infections such as pneumonia, meningitis, endocarditis, and urinary tract. ${ }^{1}$ Three decades ago, Acinetobacter baumannii infections treated with traditional antibiotics but today its show resistance to major classes of antibiotics including aminoglycosides, tetracyclines, fluroquinolones, carbapenms, cephalosporins, etc... At last year's multidrug resistance (MDR) clinical isolates have shown global distribution. ${ }^{2}$ Since Discovery and use of antibiotics, resistance to these agents has been observed. That has negative effect on the treatment of infectious disease. ${ }^{3}$ Bacteria achieve their resistance to the antibiotics through three mechanisms: (1) efflux of the antibiotic from the cell via membrane-associted pumping proteins. (2) modification of antibiotic binding target molecule such as special protein or ribosomal RNA or by reprogramming of biosynthetic pathways.(3) by modifying enzymes that selectively modified and destroyed of antibiotic activity. These mechanisms require new programming by the cell in response to the presence of antibiotics. ${ }^{4}$ Acetyl transfer, is a common mechanism for in activation of antibiotic that employed by bacteria. O-acetylation or $\mathrm{N}$-acetylation is biologically stable. The aminoglycoside antibiotics bind to the A-site of the ribosome and as e result, impire the codon-anticodon decoding mechanism and blocking of translation fidelity. Aminoglycoside antibiotics bind to $16 \mathrm{~s}$ rRNA molecule. ${ }^{5}$ Aminoglycoside acetyl transferase are member of GCN5 super family of protein include the histon acetyl transferase that are classified based on their region specificity of acetyl transfer on the aminoglycoside structure. For example AAC (3) and AAC(6) $\mathrm{N}$ acetylate aminoglycoside on the amine grope on position 3 and 6aminohexose respectively. Genes encoding these enzymes are widespread in plasmids, transposons, and integrons (Figure 1). ${ }^{6}$ In this is study we are survey present of AAC $(3,6)$ gen in Acinetobacter baumannii isolated from Iranian hospital patient.

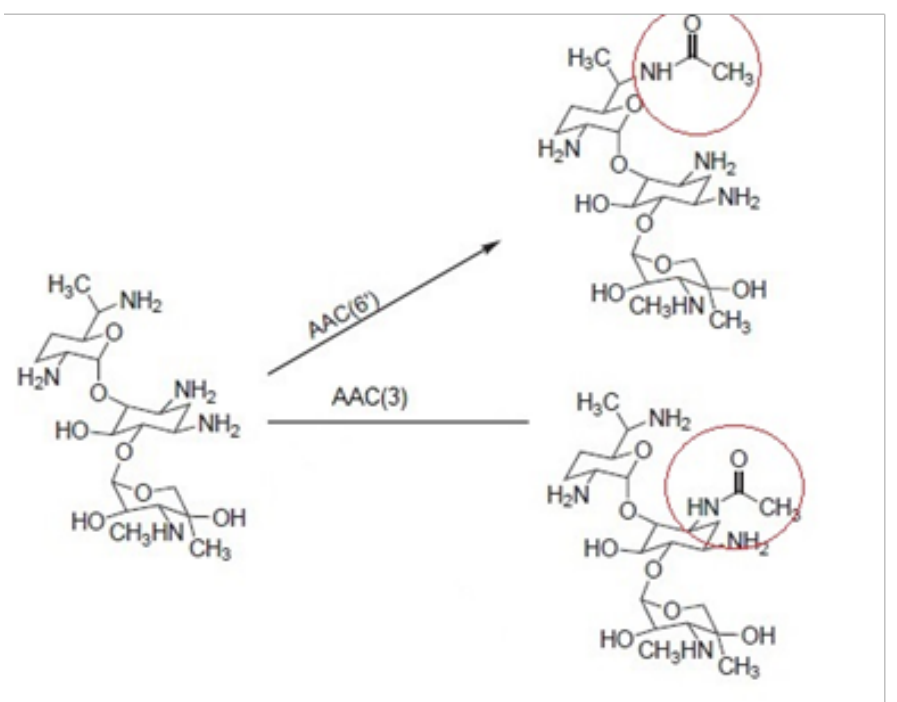

Figure I Reaction catalysed by AACs.

\section{Methods and materials}

A total of 43 non-duplicated Acinetobacter clinical isolates were collected from the Tehran hospital in 2016.Biochemical test were used for identification at the species level in 43 gram negative bacteria that had negative reaction on oxidase test and lack of lactose fermentation and TSI tests Alk/Alk. ${ }^{7-9}$ The strains were isolated from trachea $(60 \%)$ and sputum $(40 \%)$. Multi drug resistance tests carried 
out foe several antibiotics grope but in this analysis aminoglycoside (amikacin, gentamicin) resistance strains selected for study. Antibiotic susceptibility testing was performed using disc diffusion method (Kirby-bauer) on Muller Hinton agar. The criteria used were in accordance with the guidelines established by the Clinical and Laboratory Standards Institute (CLSI) ${ }^{8}$ DNA extraction carried out by gram negative DNA extraction kit. Two target gens and their primers used for PCR amplification are listed in below Table 1 .

Table I Two target gens and their primers used for PCR amplification

\begin{tabular}{|c|c|c|c|c|}
\hline Primer name & Primer sequence (5to3) & Genes & Bp & Reference \\
\hline & CGGAAGGCAATAACGGAG For & & & \\
\hline \multirow[t]{3}{*}{ aac(3)Ila } & & AAC(3lla & sz740 & 10 \\
\hline & TCGAACAGGTAGCACTGAG Rev & & & \\
\hline & TTGCGATGCTCTATGAGTGGCT For & & & \\
\hline \multirow[t]{2}{*}{$\mathrm{aac}(6) \mathrm{lb}$} & & $\mathrm{AAC}(6 \mathrm{lb}$ & 482 & II \\
\hline & CTCGAATGCCTGGCGTGTTT Rev & & & \\
\hline
\end{tabular}

PCR condition included denaturation at $94^{\circ} \mathrm{C}$ for $2 \mathrm{~min} ; 36$ cycles consisting of $94^{\circ} \mathrm{C}$ for $45 \mathrm{~s}$, annealing at $58^{\circ} \mathrm{C}$ (for aac(6)Ib) and $54^{\circ} \mathrm{C}$ (for aac(3)IIa) for $45 \mathrm{~s}$, and $72^{\circ} \mathrm{C}$ for $45 \mathrm{~s}$; and a final extension at $72^{\circ} \mathrm{C}$ for 5 mins. ${ }^{7} \mathrm{PCR}$ product electrophoresed on agarose gel $1 \%$ for aac(3)IIa and agarose $1.5 \%$ for aac(6)Ib. $100 \mathrm{bp}-1 \mathrm{kbp}$ DNA ladder was used to assess PCR product size and treatment 10min with ethtidium bromide and imaging with UV illuminator. ${ }^{9}$

\section{Result}

Acinetobacter spp isolated were recovered from 43 patient that in ICUs and some other part of hospital. The strains were isolated from trachea $(60 \%)$ and sputum $(40 \%)$. Multi drug resistance tests carried out foe several antibiotics grope. Isolated Acinetobacters pp has high resistance to all groups of antibiotics. Aminoglycoside resistance for amikacin, was $95 \%$ and for gentamicin was $93 \%$. Aac (3)IIa gene with $740 \mathrm{bp}$ was $68.4 \%$ and in gentamicin resistant strain was $66.9 \%$. aac (6)Ib gene with $482 \mathrm{bp}$ present in $76 \%$ of resistant strain (43/33) and in amikacin resistant strain was $72.2 \%$ and in gentamicin resistant strain was $70.6 \%$. Figure $2 \& 3$ show result of PCR product electrophoresis the last sample from right related to negative control and sample without band related to negative sample for Acinetobacter and band show present of gene in bacteria first column from left related to DNA ladder band.
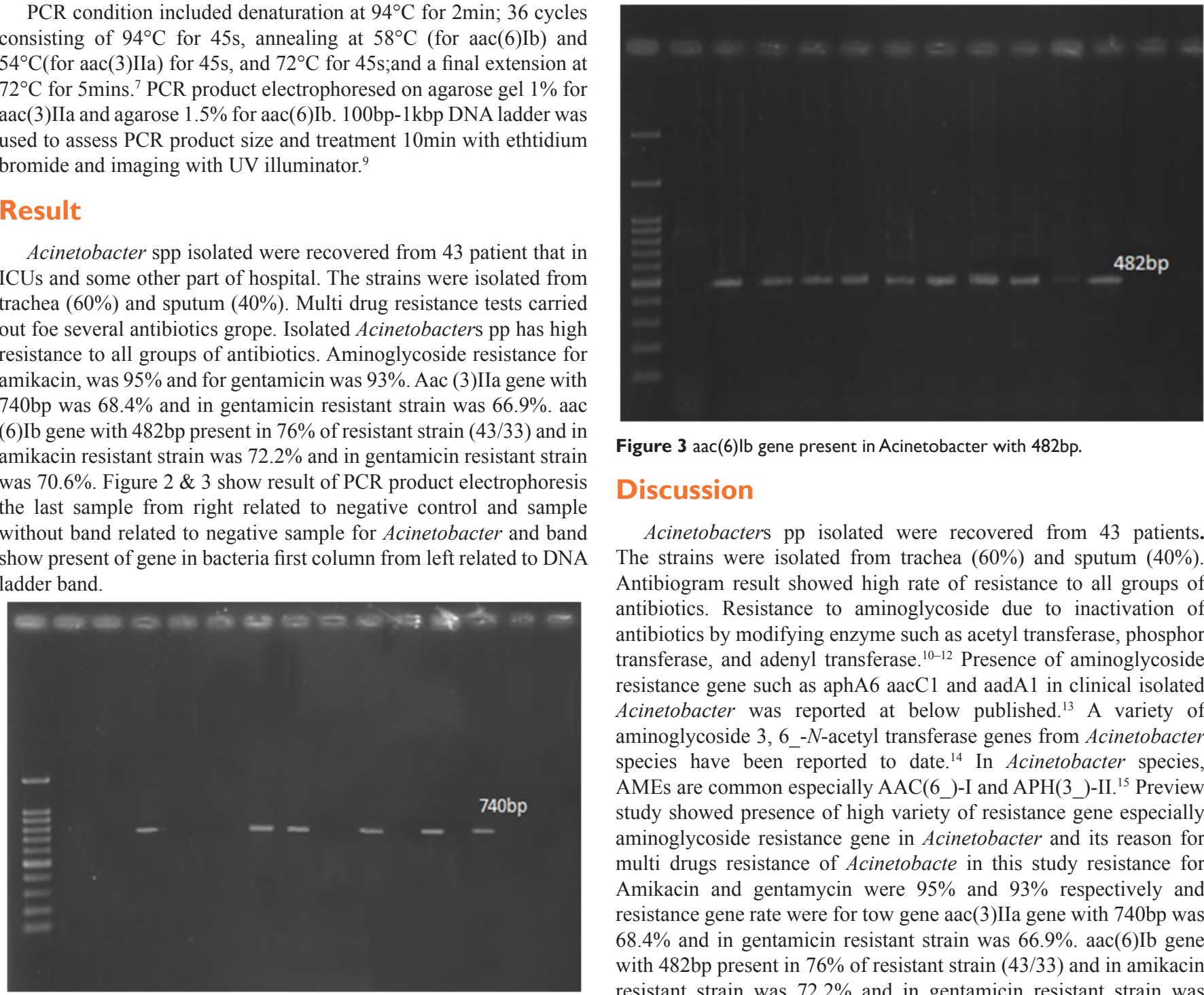

Figure $3 \mathrm{aac}(6) \mathrm{lb}$ gene present in Acinetobacter with $482 \mathrm{bp}$.

\section{Discussion}

Acinetobacters pp isolated were recovered from 43 patients. The strains were isolated from trachea $(60 \%)$ and sputum $(40 \%)$. Antibiogram result showed high rate of resistance to all groups of antibiotics. Resistance to aminoglycoside due to inactivation of antibiotics by modifying enzyme such as acetyl transferase, phosphor transferase, and adenyl transferase. ${ }^{10-12}$ Presence of aminoglycoside resistance gene such as aphA6 aacC1 and aadA1 in clinical isolated Acinetobacter was reported at below published. ${ }^{13}$ A variety of aminoglycoside 3, 6_- $N$-acetyl transferase genes from Acinetobacter species have been reported to date. ${ }^{14}$ In Acinetobacter species, AMEs are common especially AAC(6_)-I and APH(3_)-II. ${ }^{15}$ Preview study showed presence of high variety of resistance gene especially aminoglycoside resistance gene in Acinetobacter and its reason for multi drugs resistance of Acinetobacte in this study resistance for Amikacin and gentamycin were $95 \%$ and $93 \%$ respectively and resistance gene rate were for tow gene aac(3)IIa gene with 740bp was $68.4 \%$ and in gentamicin resistant strain was $66.9 \%$. aac(6)Ib gene with $482 \mathrm{bp}$ present in $76 \%$ of resistant strain (43/33) and in amikacin resistant strain was $72.2 \%$ and in gentamicin resistant strain was

Figure 2 aac(3)Ila gene present in Acinetobacter with 740bp. $70.6 \%$. Its show that other genes may be have role in Acinetobacter 
resistance for aminoglycoside. Resistance to so antibiotics perhaps the intrinsic impermeability of these outer membranes coupled with the close relationship of $A$. baumannii to the soil and aquatic environment has made it possible for these organisms to acquire highly effective resistance determinants in response to multiple challenges. ${ }^{16}$

\section{Conclusion}

It believes that the ability of this pathogen to harbor diverse genetic elements parallels the experience with $P$. aeruginosa. Genome wide analysis will provide critical insights into this ability. Wasteful use of antibiotic cause to appearance of resistance strain of bacteria to the existence antibiotics and this makes treatment difficult also the cost and duration of treatment increased.

\section{Acknowledgements}

None.

\section{Conflict of interest}

The author declares no conflict of interest.

\section{References}

1. Moradi J, Hashemi FB, Bahador A. Antibiotic resistance of Acinetobacterbaumanniiin iran: a systemic review of the published literature. Osong public health res prespect. 2015;6(2):79-86.

2. Oh yj, Song SH, Baik SH, et al. A case of fulminant community acquired Acinetobacter baumannii pneumonia in korea. Korean $j$ intern med. 2013;28(4):486-490.

3. Levy SB. The antibiotic paradox. UK: Perseus Publishing; 2002. 335 p.

4. Jackowski S. Biosynthesis of pantothenic acid and coenzyme A. Eco Sal Plus. 1996;2(2):687-694.

5. Wright GD. Bacterial resistance to antibiotics: Enzymatic degradation and modification. Advanced Drug Delivery Reviews. 2005;57(10):14511470 .
6. Vetting MW, Magnet S, Nieves E, et al. A bacterial acetyltransferase capable of regioselectiveN-acetylation of antibiotics and histones. Chem Biol. 2004;11(4):565-573.

7. Lin YC, Hsia KC, Chen YC, et al. Genetic Basis of Multidrug Resistance in Acinetobacter Clinical Isolates in Taiwan. Antimicrobial agents and chemotherapy. 2010;62(2):2078-2084.

8. Clinical and Laboratory Standards Institute Performance standards for antimicrobial susceptibility testing: fifteenth informational supplement, M100-S15. USA: Clinical and Laboratory Standards Institute; 2014.

9. Moniri R, Farahani RK, Shajani G, et al. Molecular epidemiology of aminohlycosides resistant in Acintobacter SPP. With emergence of multidrug-resistant strains. Iranian J publ Health. 2010;39(2):63-68.

10. Wu Q, Zhang Y, Han L, et al. Plasmid-mediated 16SrRNA methylase in aminoglycoside- reistantenterobactriaceae isolates in shghai, china. Antimicrobial agents and chemotrapy. 2008;53(1):271-272.

11. Over U, Gur D, Unal S, et al. The changing nature of aminoglycosiceresistence mechanisms and prevalence of newlyreconizedresistence mechanisms in turkey. Clin microbial infect. 2001;7:470-478.

12. Lambert T, Rudant E, Bouvet $\mathrm{P}$, et al. Molecular basis of aminoglycoside resistance in Acinetobacter spp. J Med Microbiol. 1997;46:731-35.

13. Shaw KJ, Rather PN, Hare RS, et al. Molecular genetics of aminoglycoside resistance genes and familial relationships of the aminoglycoside-modifying enzymes. Microbiol Rev. 1993;57(1):13863.

14. Doi Y, Wachino J, Yamane K, et al. Spread of Novel Aminoglycoside Resistance Gene aac(6 )-Iad among Acinetobacter Clinical Isolates in Japan. Antimicrob Agents Chemother. 2004;48(6):2075-2080.

15. Pitt TL, Livermore DM, Miller G, et al. Resistance mechanisms of multiresistant serotype 012 Pseudomonas aeruginosa isolated in Europe. J Antimicrob Chemother. 1990;26(3):319-328.

16. D'Costa VM, McGrann KM, Hughes DW, et al. Sampling the antibiotic resistome. Science. 2006;311(5759):374-377. 